\title{
Investigation of structure differences between archaeal and human translation initiation factor 2
}

\author{
Tarabarova A.G., Nikonova E.Y., Nevskaya N.A., Nikonov S.V., Nikonov O.S. \\ Institute of Protein Research, RAS, Pushchino, Russia \\ *alik@vega.protres.ru
}

Key words: eIF2, aIF2, protein structure, translation

Motivation and Aim: Genetic analyses of patients with MEHMO syndrome have identified a homozygous missense variant I222T in the X-linked EIF2S3 gene encoding the human (Hsa) IF2 $\gamma$ subunit which is a part of translation initiation factor 2 (e/aIF2) in eukaryotic and archaeal protein synthesis. The pathogenic mechanism of the mutation is unknown. To resolve it, we introduced the corresponding mutation (I181T) in the archaeal SsoIF2 $\gamma$ subunit, but this did not change structure of the SsoIF2 $\gamma$ and its affinity to SsoIF2 $\beta$. The analysis of the human $\beta \gamma$ interface in theoretical model has shown that such interface is less accessible to the solvent in contrast with the SsoIF2 $\beta \gamma$. Our study is aimed to investigate the effects of the introduced mutation and to specify the features of HsaIF2.

Methods and Algorithms: Mutations were introduced by QuikChange method and verified by Sanger sequencing. Recombinant proteins were synthesized using the $E$. coli BL21(DE3) strain, and purified by series of chromatography. SPR method was used to measure the kinetics of the proteins interaction in vitro.

Results: We established the effects of the studied mutations at the interaction sites between the proteins: (1) replacement of Ile181Thr in SsoIF2 $\gamma$ impairs binding to HsaIF2 $\beta$; (2) replacement of Gly197Tyr improves the interaction of SsoIF2 $\gamma$ with SceIF2 $\beta$, HsaIF2 $\beta$, and SsoIF2 $\beta$; (3) both mutations Gly197Tyr and Ile181Thr in SsoIF $2 \gamma$ improve the interaction with SceIF2 $\beta$, HsaIF $2 \beta$. These results helped us to come closer to understanding of the mechanism of HsaIF $2 \gamma$-HsaIF $2 \beta$ interaction. The stronger disrupting effect of the Ile181Thr amino acid substitution was in the interaction with the human beta-subunit. The positions of the alpha helix, which mediates interaction between the two subunits, differs in the human and the archaeal beta-subunits. In the archaeal protein it is at the N-terminal, while in humans it is in the middle of the protein. Probably these sites are unstructured in solution, and take on the alpha helix structure only after binding with the gamma-subunit. Thus, the archaeal N-terminal site has more degrees of freedom and can adapt to new conditions caused by the mutation, in contrast to the human beta-subunit site, which is located in the middle of the polypeptide chain and has more steric restrictions.

Conclusion: The study identified new potential features of the interaction between the beta- and gamma-subunit of the human translation initiation factor 2.

Acknowledgements: This study was supported by Russian Ministry of Education and Science state project AAAA-A19-119122490038-8. 